\title{
Variations
}

Variations

Revue internationale de théorie critique

$9 / 10 \mid 2007$

Les frontières de la politique

\section{Le conflit entre le pouvoir, le nombre et les armes}

Prééminence et potentialité du pouvoir chez Arendt

\section{Lucas G. Martín}

\section{(2) OpenEdition}

Journals

Édition électronique

URL : http://journals.openedition.org/variations/481

DOI : $10.4000 /$ variations.481

ISSN : 1968-3960

Éditeur

Les amis de Variations

Édition imprimée

Date de publication : 1 juin 2007

Pagination : 134-148

Référence électronique

Lucas G. Martín, «Le conflit entre le pouvoir, le nombre et les armes », Variations [En ligne], 9/10 | 2007, mis en ligne le 01 décembre 2012, consulté le 19 avril 2019. URL : http://journals.openedition.org/ variations/481; DOI : 10.4000/variations.481

Ce document a été généré automatiquement le 19 avril 2019.

Les ami•e•s de Variations 


\title{
Le conflit entre le pouvoir, le nombre et les armes
}

\author{
Prééminence et potentialité du pouvoir chez Arendt
}

Lucas G. Martín

Hannah Arendt a été accusée d'être une nostalgique de la cité grecque, accusée aussi d'un irénisme imputable à sa notion d'action opposée à la domination et à la violence. Ses détracteurs s'empressent de prétendre qu'elle a manqué de réalisme. Des disciples, qui ne lui rendent pas un meilleur hommage, réduisent sa pensée au propos politiquement correct et passe-partout du «vivre-ensemble». Quant à ceux qui ne sont ni pour ni contre, ils ont le droit de se demander à quoi bon parler de "vivre-ensemble ", d'autant que le problème réside justement dans le fait de ne pas avoir le pouvoir de "vivre-séparé » (séparé de la toute-puissance des dominants, de la corruption, des rapports autoritaires). Enfin, lorsque nos mœurs politiques sont secouées par la violence des émeutiers de banlieue - fatigués, eux, de la part qui est la leur dans l'« ensemble» du «vivre ensemble » - nous avons du mal à nous convaincre, nous et les autres, de la pertinence de penser avec Arendt. Car nous avons l'intuition que, dans cette violence exaltée extramuros, le politique est en jeu tandis que l'on sait l'opposition arendtienne entre politique et violence. Tous ces regards (pour, contre, réservés) convergent dans une seule et même question: à quoi bon revenir sur Arendt? Il faut, tout d'abord, savoir qu'Arendt a été confrontée de son vivant à ce type de regards ainsi qu'à des réalités aussi frappantes que celles que nous connaissons. Il faut également se méfier aussi bien des critiques des détracteurs au nom du réalisme que des éloges des porte-parole de la solution du vivreensemble. Il n'y a pas là deux lectures opposées d'Arendt, mais deux appels à l'abandon de sa pensée. Les uns et les autres partagent un refus d'Arendt, dénonçant le fait que l'action politique deviendrait soit impuissante à l'égard de la réalité, soit superflue car nous sommes tous invités à délibérer ensemble afin d'arriver à un consensus (et voilà, personne ne peut se dire exclu!). Méfions-nous de cet accord entre frères ennemis exigeant l'abandon d'une pensée qui a revendiqué autant les révolutions que la désobéissance civile et qu'on peut lire aussi comme une pensée du "vivre-contre». C'est cet aspect du vivre-contre que nous tâcherons de récupérer, cette récupération étant 
possible en relevant les ambiguités qui sont au sein de la conception arendtienne du pouvoir. Car, chez Arendt, il se peut qu'on lise parfois que le pouvoir dépend du nombre, parfois qu'il en est indépendant; de même qu'un petit nombre peut être aussi puissant qu'un grand nombre, mais que, dans une certaine mesure, le pouvoir du petit nombre dépend en fait de la quantité de ceux qui partagent une opinion; encore, que la violence est indépendante du nombre et opposée au pouvoir, mais que, en dernier ressort, elle dépend des deux.

2 Dans les pages qui suivent, on analysera ces ambiguïtés du concept de pouvoir dans l'œuvre d'Arendt, en particulier celles qui relèvent des rapports du pouvoir avec le nombre et la violence. Deux hypothèses seront discutées : d'une part, celle de la légitimité d'une problématique chez Arendt autour de la notion de pouvoir et, d'autre part, celle qui viendrait à répondre à une telle problématique: ce serait la notion d'un conflit à l'intérieur du pouvoir, un conflit constant du pouvoir contre le pouvoir, ce qui peut résoudre lesdites ambiguïtés. La première hypothèse sera déployée au long du texte pour aboutir vers la fin aux six thèses sur le pouvoir, dans lesquelles j'expose la deuxième hypothèse. Or, la réponse ne résout pas les ambiguïtés, faute de quoi elles sont transposées en la figure d'un conflit de potentialités à l'intérieur du pouvoir - et non pas en une quelconque univocité. Autrement dit, on soutiendra que les ambiguïtés théoriques font signe des tensions inhérentes à l'expérience du pouvoir. Notre propos sera ainsi développé en deux temps. Dans un premier temps, on proposera une définition du pouvoir selon Arendt en distinguant ses trois dimensions - pluralité, potentialité et prééminence -, lesquelles nous serviront lors de l'examen des ambiguïtés qui se présenteront au fur et à mesure. Avant de passer aux ambiguïtés, on établira aussi l'opposition que fait Arendt entre pouvoir et violence. L'analyse des ambiguïtés à l'égard des différentes dimensions du pouvoir va nous conduire à un rapprochement entre ces deux concepts. Certes, il apparaitra qu'un pouvoir, toujours présent "derrière " la violence, exige une pensée complexe de la différence essentielle entre ces deux concepts. C'est pourquoi ce problème irrésolu sera finalement examiné à la lumière de la question du pouvoir du petit nombre. On y sera confronté à l'enjeu ambigu entre potentialité et prééminence à partir des citations qu'Arendt fait d'une phrase de Mirabeau. On posera alors la question de savoir s'il existe aussi un pouvoir « derrière » le pouvoir, un pouvoir prééminent. Finalement, dans les conclusions, nous proposerons une réponse possible aux ambiguïtés, et notre deuxième hypothèse, qui sera déployée en six thèses sur le pouvoir, soulignera notamment l'existence, inhérente au pouvoir, d'un conflit de potentialités.

\section{Le pouvoir contre la violence}

Il est possible de suivre dans toute l'œuvre d'Arendt la trace de ses efforts pour élaborer une définition claire et distincte du pouvoir. Pour une première définition, on se tiendra cependant aux deux textes où elle élabore ses définitions du pouvoir: La Condition de l'homme moderne, notamment, le paragraphe intitulé «La puissance et l'espace de l'apparence » du chapitre V (consacré à l'action), et l'essai "Sur la violence », publié dans Du mensonge à la violence ${ }^{1}$. Sans nul doute, ces textes font preuve de la continuité et de la cohérence avec lesquelles Arendt oppose le pouvoir à la domination, celle-ci étant entendue comme rapport de commandement et obéissance, et celui-là renvoyant à l'expérience de l'isonomie. De même, on peut constater que, dans ces textes, elle rapporte 
le pouvoir à la liberté, particulièrement à partir de sa définition de l'action comme nouveau commencement, sans précédent, où « il n'y a pas d'être derrière l'agir »².

Dans «Sur la violence », Arendt soutient que "[le] pouvoir correspond à l'aptitude de l'homme à agir, et à agir de façon concertée. Le pouvoir n'est jamais une propriété individuelle; il appartient à un groupe et continue à lui appartenir aussi longtemps que ce groupe n'est pas divisé3. » Quelques années auparavant, dans La Condition de l'homme moderne, elle avait déjà lié le pouvoir au fait « que des hommes s'assemblent dans le mode de la parole et de l'action", donnant lieu à un espace d'apparence qui "précède par conséquent toute constitution formelle du domaine public et des formes de gouvernement » et qui dure autant qu'ils demeurent réunis par l'action et la parole et jusqu'à ce qu'ils se dispersent ${ }^{4}$. "C'est la puissance, dit Arendt, qui assure l'existence du domaine public, de l'espace potentiel d'apparence entre les hommes agissant et parlant ${ }^{5}$ . » Autrement dit, autant il y a d'espace d'apparence, autant il est de la puissance et, viceversa, autant on est puissants, autant il est possible d'apparaître. De ceci, nous pouvons dégager trois dimensions caractéristiques du concept de pouvoir. D'abord, il y a un caractère pluriel ou une dimension de pluralité : le pouvoir est une capacité collective - de " plusieurs » et non pas d'un seul individu - coïncidant avec la pluralité qu'Arendt jugeait être la condition de l'action (au double sens du terme, sans laquelle on ne peut pas et à partir de laquelle on peut). C'est en ce sens que le pouvoir semble être le revers de l'action, son autre visage coextensif.

Ensuite, le pouvoir a un caractère potentiel, ou une dimension de potentialité, qui constitue l'espace d'apparence pour l'acteur. Cet espace de potentialité coïncide avec l'assemblement du nombre et s'actualise en tant que potentialité pour autant que la pluralité demeure réunie. Arendt affirme que «[la] puissance est toujours [...] une puissance possible », une puissance potentielle, a power potential, s'actualisant en tant que potentialité et ne pouvant ni se matérialiser, ni se posséder, ni s'emmagasiner. C'est pourquoi le rapport entre potentialité et action, entre le pouvoir et l'acteur, ne peut pas être figuré selon l'idée de succession ou de séquence (selon laquelle la puissance précéderait l'acte qui l'actualise) mais comme simultanéité. En ce sens, le pouvoir continue d'être le revers de l'action puisque, de surcroît, on n'a notion de son existence qu'à travers l'action, car tout pouvoir qui ne se manifeste pas se perd ${ }^{6}$. Enfin, à un pouvoir à plusieurs et ouvrant le champ des potentialités, il faut ajouter une troisième dimension, celle de la prééminence du pouvoir, la "précédence» du pouvoir vis-à-vis de toute organisation « formelle » d'un régime ou d'un gouvernement. Il faudra certes distinguer cette prééminence/précédence d'une précédence chronologique : il s'agit, dit Arendt, de la «prééminence fondamentale du pouvoir sur la violence ${ }^{7}$ ». Cet aspect se déduit de ceux qui précèdent, puisque seul le pouvoir engendre du pouvoir et qu'il existe autant qu'il existe du nombre, il confirme la prétention arendtienne, mentionnée au début, de différencier le concept de pouvoir de celui de domination, prétention illustrée à plusieurs reprises par Arendt avec la phrase de Madison qui dit que «tout gouvernement repose sur l'opinion" ${ }^{8}$.

6 Pluriel, potentiel et prééminent, le pouvoir apparait ainsi chez Arendt investi des traits qu'on ne saurait facilement reconnaître dans n'importe quelle situation politique du moment. Toutefois, si puissance il y a, si l'on ose encore conjuguer le verbe " pouvoir ", il nous faudra en examiner les raisons dans ces trois dimensions. Ceci sans réduire le pouvoir à une violence nous permettant parfois d'atteindre des buts particuliers et dont la pratique devient plus efficace quand un plus grand nombre s'y engage. Certes, on peut, 
moyennant la violence, mener à bien nos intérêts de groupe. Cependant, Arendt refuse de voir de la potentialité dans l'expérience violente.

Or, l'insistance sur cette distinction ne règle apparemment pas l'affaire puisque, quelques années plus tard, elle revient au cœur des réflexions d'Arendt. En 1970 paraît « Sur la violence », où elle s'applique à différencier, entre autres, les concepts de " pouvoir » et de " violence ». C'est alors que l'auteur s'exprime avec une clarté qui ne devrait laisser place à aucun doute: «Il ne suffit pas de dire que, dans le domaine politique, il ne faut pas confondre pouvoir et violence. Le pouvoir et la violence s'opposent par leur nature même ; lorsque l'un des deux prédomine de façon absolue, l'autre est éliminé9."

Cette opposition est étayée par trois arguments. D'abord, la violence manque d'essence propre en vertu de sa dépendance par rapport au but qui l'exige comme moyen et qui lui sera extérieur par définition. Elle nécessite ainsi des justifications, de même que n'importe quel moyen. Le pouvoir, en revanche, se passe de cette condition, parce que, tout en coïncidant avec l'existence du rassemblement pluriel de la communauté, il est le moyen autant que la fin, de sorte qu'il ne saurait être intelligible sous le schéma moyenfin. Ensuite, la violence ne peut pas avoir pour but la génération du pouvoir, parce que son caractère instrumental dissout le lien que l'agir-des-uns-avec-les-autres établit et qui est le propre du pouvoir. "Seul le pouvoir en tant que potentialité - seule l'action concertée des hommes - engendre du pouvoir ${ }^{10}$. » Enfin, pouvoir et violence sont opposés en regard de leur rapport au nombre: tandis que le pouvoir dépend du nombre, la violence en est indépendante dans la mesure où elle est plutôt attachée à des instruments matériels avec lesquels elle multiplie la force d'un groupe peu nombreux, voire d'un seul individu $^{11}$. C'est ainsi qu'un seul peut, par exemple, arriver à s'imposer sur un grand nombre d'hommes.

9 En somme, tandis que le pouvoir est essentiel (fait du sens), engendre plus de pouvoir et dépend du nombre, la violence est instrumentale, simple moyen détruisant des parties du monde et pouvant se passer du nombre. Or, nous pourrions nous demander quel genre de potentialité est celle qui s'appuie sur le nombre, ou quelle efficacité reconnaître à la violence d'un seul individu isolé par une multitude refusant d'obéir. Serait-ce que le pouvoir, se manifestant par la force du nombre, l'emporte sur la violence du petit nombre? Ne s'agit-il pas d'une confrontation entre les ressources d'oppression des dominants et la quantité d'hommes rassemblés pour s'y opposer, le résultat provenant de la concurrence entre le nombre et les instruments?

\section{Indépendance du pouvoir par rapport au nombre (sa dimension potentielle)}

10 Dans le paragraphe consacré à définir et à distinguer les notions de pouvoir et de violence, et apparemment à l'encontre de la dimension " plurielle » du pouvoir évoquée plus haut, Arendt énonce l'indépendance du pouvoir à l'égard du nombre. Le fragment se trouve dans le paragraphe déjà mentionné du chapitre $\mathrm{V}$ de CHM: «La puissance est toujours, dirions-nous, une puissance possible, et non une entité inchangeable, mesurable et sûre, comme l'énergie [force] ou la force [strength]. Tandis que la force est la qualité naturelle de l'individu isolé, la puissance jaillit parmi les hommes lorsqu'ils agissent ensemble et retombe dès qu'ils se dispersent. En raison de cette particularité que la puissance partage avec tous les possibles [potentialities], qui peuvent seulement 
s'actualiser et jamais se matérialiser pleinement, la puissance est à un degré étonnant indépendante des facteurs matériels, nombre et ressources ${ }^{12}$. »

Voilà ce double rapport du pouvoir au nombre. Comment se pourrait-il que le pouvoir soit, en même temps, dépendant et indépendant du nombre? La réponse serait, en principe, simple : il y aurait deux modes d'être du nombre, l'un, qualitatif et pluriel, l'autre, quantitatif et homogène. Le premier mode désigne un être pluriel, un mode d'être où le pouvoir généré surgit dans l'espace entretenu, de sorte que, par un double mouvement d'assemblement et de séparation, les "plusieurs » composant cette pluralité conservent un espace "entre » eux (inter-esse) et empêchent la constitution d'une unité homogène. Ce mode d'être du nombre, pour le dire avec des termes proches de ceux de Jacques Rancière, serait « incomptable ${ }^{13}$ ». Le mode d'être quantitatif (mesurable) et homogène désigne, en revanche, une quantité de choses ou d'êtres pouvant être comptés au même titre que sont comptés la population d'une ville, les membres d'un parti ou l'importance d'une émeute. Il s'agit d'une homogénéité dont la mesure ou la valeur change en fonction du nombre des éléments qui la composent. À la différence du pouvoir politique, sa "puissance », ou plus précisément, sa force, dépend de leur nombre.

Le pouvoir politique, en tant que potentialité, demeure ainsi le propre de la pluralité rassemblée, c'est-à-dire qu'il existe dans la mesure où il continue à « être un potentiel de rassemblement qualifiant une communauté et non une puissance quantifiable définissant une entité14 ${ }^{14}$. Il en résulte que tant le pouvoir que la liberté politique résident dans un type de relation et non dans la quantité de ceux qui y entrent en rapport. Un petit nombre peut être aussi libre que puissant. En d'autres termes, le pouvoir est aussi dépendant du mode pluriel du nombre qu'il n'est indépendant de son mode quantitatif, matériel et homogène. Or, il faut admettre que cet éclaircissement à l'égard du rapport du pouvoir au nombre tranche une distinction entre le pouvoir et la force du nombre ${ }^{15}$, mais non pas entre pouvoir et violence, car les instruments propres à la violence ne sont pas encore en jeu. Il nous faut donc nous poser maintenant cette question du nombre à l'égard de la violence.

\section{Quelle indépendance de la violence vis-à-vis du nombre?}

13 Nous suggérions tout à l'heure qu'il fallait tenir compte du rapport au nombre pour donner une définition de la violence et du pouvoir. En effet, la première pouvait se définir par son indépendance par rapport au nombre, tandis que le deuxième dépendait du nombre au sens qualitatif tout en restant indépendant du nombre au sens quantitatif. Or, si l'on continue à affirmer l'opposition entre violence et pouvoir, ces deux concepts doivent à tout le moins être opposés par leur rapport à l'un des deux modes d'être du nombre, soit qualitatif soit quantitatif. Dans quel sens la violence est-elle indépendante du nombre ? D'après ce qu'on a constaté jusqu'ici, la violence est par définition dispensée du mode d'être quantitatif du nombre tandis qu'elle s'oppose à son mode d'être qualitatif, soit au pouvoir. En effet, pour qu'on puisse conserver son opposition au pouvoir, la violence devra tenir aussi un rapport d'indépendance vis-à-vis de ce mode d'être dont le pouvoir est, par définition, dépendant. De même, quitte à défaire le caractère instrumental de la violence, son indépendance à l'égard du mode quantitatif du nombre doit aussi être conservée. Somme toute, elle serait doublement indépendante (elle devrait 
l'être). Pourtant, dans "Sur la violence ", on lit une phrase un peu troublante juste au moment où Arendt est censée avoir tranché la différence entre pouvoir et violence : « En fait, une des différences les plus caractéristiques qui permettent de distinguer le pouvoir de la violence est que le pouvoir a toujours besoin de s'appuyer sur la force du nombre, tandis que la violence peut s'en passer, dans une certaine mesure [up to a point], du fait que pour s'imposer elle peut recourir à des instruments ${ }^{16}$. »

A lire la réserve - «dans une certaine mesure [up to a point] » - toutes nos certitudes à l'égard d'une définition tranchée de la violence et du pouvoir ainsi que de leur opposition inexorable, sont remises en cause. En effet, toute introduction du nombre, quelque marginale qu'elle soit dans le concept de violence, modifie l'idée que nous nous faisions de son expérience. D'une part, pour autant que l'on admette le nombre au sens quantitatif, même si ce n'est que "dans une certaine mesure", on se rapproche de l'expérience de l'énergie, ou force [force], et peut-être de celle de la force individuelle, ou vigueur [strength]. On perdrait ainsi la substance claire et distincte d'une violence définie par un caractère instrumental qui viole l'être naturel des choses, la force inhérente de tout ce qui est. D'autre part, si l'on admet la moindre dépendance par rapport au mode d'être pluriel du nombre, on mène la violence au domaine politique des affaires humaines où les instruments n'ont pas droit de cité. Quel est alors le sens de cette réserve introduite par Arendt? Et quel est le rapport entre violence et pouvoir, entre le recours aux instruments et le potentiel d'une simple pluralité, enfin entre domination et liberté ?

\section{La prééminence du pouvoir : "les armes elles-mêmes changent de mains »}

Dans le même texte, nous découvrons que le rapprochement entre violence et nombre n'était pas un simple glissement. Arendt y expose l'argument de la dépendance de la violence et $\mathrm{du}$ gouvernement par rapport au pouvoir: "Les moyens de la violence deviennent inutiles quand on n'obéit plus aux ordres, et le facteur décisif n'est plus alors une question d'obéissance au commandement, mais un problème d'opinion: celle du nombre plus ou moins grand de ceux qui sont du même avis. Tout dépend du pouvoir qu'il y a derrière la violence. [...] L'obéissance civile [...] n'est que la manifestation extérieure du soutien et du consentement ${ }^{17}$. $"$

Ce double mouvement par lequel, d'une part, « tout dépend du pouvoir qu'il y a derrière la violence » et, d'autre part, le pouvoir dépend du nombre de ceux qui partagent telle opinion, nous mène à conclure que la violence dépend du pouvoir et, en conséquence, du nombre dans son mode d'être pluriel. C'est là que la dimension de prééminence, dont la phrase de Madison «tout gouvernement repose sur l'opinion» sert d'exemple, va évidemment à l'encontre de l'opposition entre pouvoir et violence. Sachant que l'un et l'autre coïncident dans leur indépendance vis-à-vis du nombre au sens quantitatif, il ne nous restait qu'à les opposer au nombre au sens qualitatif ou pluriel. Faute de quoi, Arendt perdrait ladite opposition. Pourtant, toutes ces prémisses sont ébranlées dès lors qu'on constate que la violence dépend aussi du pouvoir et du nombre au pluriel.

17 Voyons d'un peu plus près la nature de ce pouvoir derrière la violence, de ce privilège que gagne la dimension de prééminence du pouvoir lorsqu'on a affaire à la violence en politique. Dans "Sur la violence", Arendt s'attaque aussi à cette prééminence de la prééminence. Elle remarque par exemple que l'inégalité des moyens de violence entre 
l'État et n'importe quel groupe insurgé a toujours été si grande que ni le progrès technique consacré à l'amélioration des armes de l'État, ni les manuels de tactiques révolutionnaires, ne pourraient jamais empêcher le triomphe du premier sur le second. Or, ce constat sans réserve impliquerait que toute révolution est impossible, du moins si l'on parle d'une révolution «d'en bas ». Mais Arendt y dénonce un argument incomplet, car la réalité politique ne saurait être résumée à de simples affrontements entre violences. Il existe une erreur fondamentale, souligne-t-elle, à croire, comme le croyaient de nombreux révolutionnaires professionnels, que les révolutions peuvent être " réalisées ", que la violence peut créer un nouveau pouvoir et que, en conséquence, celle des armes est la bataille décisive. C'est l'idée contraire qui s'impose: c'est dans le domaine du pouvoir où - pour employer des termes sur lesquels nous reviendrons - se renversent les situations, car "les armes elles-mêmes changent de mains": "Dans une confrontation où la violence seule s'oppose à la violence, le gouvernement a toujours bénéficié d'une supériorité absolue, mais cette supériorité n'existe que pour autant que la structure du pouvoir gouvernemental demeure intacte - c'est-à-dire aussi longtemps que l'on obéit aux ordres et que l'armée, ou les forces de la police, sont prêtes à faire usage de leurs armes. Quand ce n'est plus le cas, la situation se renverse brusquement. Non seulement la rébellion n'est plus réprimée, mais les armes elles-mêmes changent de mains ${ }^{18}$ ».

Ici, quoique Arendt développe son argumentation qui vise à montrer que le caractère distinctif des révolutions est tout autre que celui de la violence qui y tient place, elle opère en même temps un déplacement par lequel un pouvoir toujours prééminent ne peut plus être compris en termes de jeu à somme nulle. Même un régime totalitaire a besoin lui aussi d'une base de pouvoir : « Même le chef d'un régime totalitaire, dont la torture est le premier instrument de gouvernement, a besoin d'une base de pouvoir : la police secrète et son réseau d'indicateurs ${ }^{19}$."

19 Nul doute que, du point de vue de la violence politique, on vérifie ainsi l'importance de ce qu'on a différencié comme la troisième dimension du pouvoir : sa prééminence. Les armes changent de mains, c'est-à-dire que les hommes changent d'opinion. Idée déjà évoquée avec la référence à Madison, elle est aussi reprise par une autre voie par Arendt dans tous les exemples qu'elle extrait de l'histoire révolutionnaire: depuis les insurrections de Hongrie et de Pologne en 1956 jusqu'à Mai 68 et aux émeutes de 1969 à Berkeley. En effet, c'est dans les moments révolutionnaires qu'il est possible de voir, ici et là, des exceptions aux règles, des acteurs qui ne coïncident pas avec leurs intérêts de groupe ou de classe et des sujets qui semblent n'être liés à aucune structure, qu'elle soit matérielle ou symbolique.

Or, si tous ces exemples historiques renvoient à la nature du pouvoir au moment où il brille grâce aux actions, c'est-à-dire, à sa dimension de potentialité, nous voulons envisager une autre dimension, moins éclatante, celle de la prééminence d'un pouvoir sous-jacent (derrière) et non actif. Qu'en est-il de ce pouvoir derrière la violence dont celleci dépend dans une certaine mesure? Comment se peut-il que, eu égard aux arguments examinés, la violence ne semble pas être si éloignée du pouvoir qu'on l'a considéré au début? Autrement dit, par ce passage d'une opposition entre pouvoir et violence à une prééminence du premier par rapport à la seconde, nous trouvons légitime de nous interroger sur les conséquences qui en découlent sur la notion de pouvoir. Se peut-il qu'il y ait une prééminence du pouvoir par rapport au pouvoir lui-même, c'est-à-dire, existe-til une sorte de méta-pouvoir aussi bien derrière le pouvoir que derrière la violence? 


\section{Six thèses autour du pouvoir}

21 À titre récapitulatif, voyons les ambiguïtés qui se sont présentées au long de notre analyse. D'abord, celle de la dépendance/indépendance du pouvoir en fonction du nombre, à partir de laquelle on a distingué deux modes d'être du nombre, l'un pluriel, l'autre quantitatif (première ambiguité). Ensuite, nous avons repris cette question à propos de la violence. Dans ce cas, quoiqu'on s'attendait à constater une double indépendance de la violence vis-à-vis des deux modes d'être du nombre (d'un côté parce qu'elle s'exerce par des instruments et, de l'autre, parce qu'elle brise toute pluralité et s'oppose au pouvoir), nous avons trouvé, en revanche, une réserve importante car, selon Arendt, la violence est indépendante du nombre dans une certaine mesure, parce qu'il y a toujours un pouvoir derrière la violence. En continuant dans cette voie, nous avons fini par remarquer un rapprochement du pouvoir vis-à-vis de la violence qui serait solidaire d'une hiérarchisation des dimensions du pouvoir élevant la dimension de prééminence au premier rang et reléguant la potentialité au deuxième (deuxième ambiguïté). Finalement, cette prééminence de la prééminence a été mise à l'épreuve du pouvoir lui-même, afin de découvrir si, lorsque la potentialité est présente, il lui faut, à elle aussi, un pouvoir derrière , comme si l'on disait qu'il existe un pouvoir derrière le pouvoir. C'est ainsi qu'on a examiné le cas d'un petit nombre d'hommes agissant et qu'on a abouti aux citations aporétiques de Mirabeau (troisième ambiguité). Dans les six thèses qui suivent, je tâcherai de présenter des arguments qui répondent aux ambiguïtés demeurées jusqu'ici sans réponse.

1. Les oppositions conflictuelles du pouvoir ne sauraient être lues en termes de force ou de violence. De même que l'on distingue deux modes d'être du nombre, de même il faudra distinguer deux modalités de conflit dépendantes de l'intervention ou de la nonintervention du pouvoir ${ }^{20}$ : l'un «singulier » ou extra-politique, l'autre "pluriel » ou de pouvoir. En vertu de la nature du pouvoir, dans un conflit ou un affrontement auquel il participe, et pour autant qu'il ne soit pas détruit, il y a une génération de pouvoir (le pouvoir engendre le pouvoir), de potentialité, définissant un mode particulier de conflit. C'est pourquoi le pouvoir, lorsqu'il intervient dans un conflit violent, peut parvenir à ce que «les armes elles-mêmes changent de mains ». En effet, puisque le pouvoir ne dépend ni du nombre ni des ressources matérielles, dans une confrontation avec la violence, le pouvoir peut renverser la situation même si ceux qui soutiennent ce pouvoir sont peu nombreux et sans moyens. Ensuite, puisque le pouvoir ne se confronte pas avec les mêmes « armes » que la violence, le conflit entre les deux, entre pouvoir et violence, ne saurait être défini comme un "combat singulier", comme s'il s'agissait d'une simple "lutte », de même que le résultat du conflit ne peut être que la victoire ou la défaite.

2. Le pouvoir ne "s'impose" ni ne "vainc" dans un conflit; il ne "domine " ni ne « force » son adversaire mais il renverse des situations, c'est-à-dire qu'il change le monde. En effet, c'est parce qu'on ne peut se l'approprier ni l'emmagasiner, qu'il n'existe pas quelqu'un (un sujet ou «qui ») de puissant qui l'emporterait sur ses opposants grâce à son pouvoir. De même, on ne peut pas dire du pouvoir qu'il est « un » et singulier, qu'il est le pouvoir, parce que le pouvoir est toujours pluriel, dans ses rapports et dans ses conflits. En effet, il n'est pas possible de distribuer le pouvoir, ni de le réifier, ni de le traiter au même titre que la force. En conséquence, c'est une erreur de dire que le pouvoir de " $\mathrm{A}$ " s'oppose à celui de « B » ou que le pouvoir « $\mathrm{A}$ » s'oppose directement à la violence ou à la 
force «B ». C'est pourquoi dire que le pouvoir « vainc ", c'est subjectiver une chose qui ne dépend jamais d'un sujet particulier, ainsi que dire qu'il «s'impose » n'est rien d'autre que dire d'une manière métaphorique qu'une situation est renversée. Seule ainsi devient compréhensible l'idée d'un conflit entre pouvoir et violence où le résultat dépend de la prééminence du pouvoir, du pouvoir qu'il y a derrière la violence, ce qui augmente la potentialité du pouvoir. Du fait que ce «derrière» n'est pas précédent au sens chronologique, mais qu'il indique la permanence de la potentialité, le pouvoir ne doit pas non plus être pensé comme quelque chose d'extérieur à la violence politique, car on éliminerait du même coup la potentialité de renverser des situations, c'est-à-dire, la possibilité que les mêmes hommes violents changent d'opinion, refusent d'obéir à un ordre de répression, laissent tomber les armes ou les retournent dans d'autres directions ${ }^{21}$.

3. La prééminence du pouvoir correspond à l'expérience qu'on fait du pouvoir potentiel de renverser des situations en tant que nous sommes spectateurs et non pas acteurs. C'est, pour ainsi dire, l'un des termes dont on dispose pour rendre compte de la potentialité une fois qu'elle est finie, soit parce qu'elle s'est perdue sans se manifester, soit parce que les actions ont été déjà accomplies. Sans un effort d'interprétation pour découvrir cette prééminence, nous risquons de céder à une description de l'histoire, comme si celle-ci était le résultat nécessaire de processus causals et de traiter les conflits de pouvoir par analogie avec les conflits de violence ou de force (c'est-à-dire, en subjectivant ou en incarnant le pouvoir dans des acteurs). C'est le risque de devenir " prophètes du passéz ${ }^{2}$ ». En effet, on ne connaît l'histoire qu'une fois qu'elle est finie, grâce au récit d'un narrateur qui, à l'égard des paroles et des prouesses qu'il raconte, est un spectateur passif, sa tâche consistant à raconter ce qui a été en partant de la base qu'il n'existe plus de potentialitée ${ }^{33}$. Certainement, le narrateur ou l'historien pourra souligner le caractère surprenant des événements lorsque, par exemple, le petit nombre ou les démunis l'emportent sur le grand nombre ou sur un groupe bien pourvu de moyens. Mais, là encore, cette idée de triomphe reste insuffisante pour rendre compte de la nature du pouvoir. En effet, il est difficile d'éviter, au moment de raconter une histoire, qu'un conflit de pouvoirs ne ressemble trop à un conflit violent et à des combats singuliers. Arendt elle-même glisse maintes fois vers une terminologie proche de la précédence chronologique. La notion de prééminence d'un pouvoir toujours derrière nous prévient de l'oubli de la potentialité et, notamment, lorsque celle-ci n'a laissé aucune trace visible dans des grands exploits, lorsque le fort a triomphé sur le faible et que la violence a écrasé le pouvoir ${ }^{24}$. Le narrateur verra à la fin de chaque histoire s'il aura à raconter l'histoire éclatante de la potentialité ou si, en revanche, il est forcé de chercher parmi les décombres de la bataille le « trésor perdu » de la prééminence du pouvoir.

4. La violence en politique n'est point pleinement violence. Au fur et à mesure que la violence accède à l'espace d'apparence des affaires humaines, elle perd sa nature muette et se contamine : elle acquiert la visibilité propre de tout ce qui se passe entre les hommes et elle commence à avoir besoin de mors pour justifier sa propre existence ${ }^{25}$. En d'autres termes, la violence subit une transformation en se rendant " politique » et elle reçoit de cet espace public ses caractères principaux : irréversibilité, imprévisibilité, possibilité de commencer quelque chose ${ }^{26}$. Ceci implique un potentiel de renversement que le pouvoir peut opposer à la violence. Quand on parle d'un pouvoir qui est derrière ou de la base de pouvoir de la violence, on doit toujours entendre qu'on a affaire à une violence qui est déjà 
une violence politique, c'est-à-dire, une violence qui s'est affaiblie du fait de son irruption dans l'espace des « affaires humaines ».

5. Dire que le pouvoir prédomine ou prime signifie que le conflit perdure. Nous ne saurions reconnaître le «triomphe » du pouvoir que dans la mesure où, paradoxalement, le conflit subsiste, c'est-à-dire, en tant que le pouvoir ne devient pas un pouvoir unique $(\mathrm{Un})^{27}$. Ceci n'est possible que dans la mesure où le pouvoir siégeant "derrière" l'opposant continue à exister et que le conflit ne se résout pas. Seulement les guerres et les combats singuliers finissent par des victoires des uns sur les autres - seules dans les guerres totales et les génocides, les défaites sont définitives et irrévocables. C'est ainsi que, dans la lecture que nous proposons d'Arendt, l'élimination du conflit est un résultat plus proche de la violence ou de la force que du pouvoir. Un pouvoir « victorieux » sera toujours un pouvoir qui ne triomphe pas sur un autre pouvoir. Tout au plus, pourrait-il diluer la violence qui accompagne cet autre pouvoir et qui menace tout pouvoir. Or, comme cela a été mis en valeur, cette primauté du pouvoir n'est possible qu'en vertu du pouvoir qui se tient derrière la violence, du jeu continu du pouvoir contre pouvoir. Un pouvoir qui détruit un autre pouvoir est, en réalité, un pouvoir violent et, en tant que tel, il met en danger sa propre potentialité28. C'est en ce sens-là que doit être interprétée la fameuse phrase qu'Arendt emprunte à Montesquieu, "seul le pouvoir arrête le pouvoir ", idée à laquelle elle ajoute: "Sans le détruire, sans mettre l'impuissance à la place du pouvoir ${ }^{29}$.» En conséquence, le pouvoir entretient un conflit indirect contre la violence : d'une part, parce que les armes sont différentes et que le pouvoir joue aussi son jeu "derrière ", d'autre part, parce qu'il s'agit de deux modes d'être et d'apparaître. C'est comme si le pouvoir ne se confrontait jamais à la violence tout court, mais qu'il menait en parallèle un conflit où le pouvoir se confronte au pouvoir. Ainsi, le pouvoir demeure autant que cette pluralité de pouvoirs persiste dans la controverse.

6. Dix hommes agissant ensemble peuvent renverser des situations parce qu'ils ne sont pas seuls, parce que le pouvoir ne dépend pas d'eux seuls, et parce qu'ils ne sont pas seulement un groupe. Dès lors que nous parlons d'un groupe qui s'impose, nous nous sommes déjà mis en position de spectateur. Notre regard suppose que le conflit de potentialités du pouvoir a été surmontée ${ }^{30}$. En revanche, si nous cherchons une approche du pouvoir, il faudra ne pas s'intéresser au groupe en tant qu'unité mais au monde, à l'espace " entre ", à l'interesse, que ce soit en considérant à l'intérieur du groupe (de sorte qu'on n'est plus face à une unité mais face à la pluralité de ses membres), soit en regardant les rapports du groupe avec d'autres groupes. Du point de vue de la praxis, ces dix hommes agissant ensemble ne constituent pas un groupe et ne sont pas définitivement limités au nombre de dix : ils sont au sein d'une potentialité en vertu de laquelle ils peuvent apparaître et, de même qu'ils ne gouvernent pas leur action, de même ils ne maîtrisent pas le nombre qu'ils constituent en tant qu'acteurs. En effet, de même que, concernant l'action, les acteurs ne peuvent pas en définir les limites, de même, ils ne gèrent pas qui ils sont ni s'ils sont nombreux ou pas. Du fait qu'«il n'y a pas d'être derrière l'agir ", leur nombre est aussi en jeu. C'est pourquoi toute action comporte un risque. En d'autres termes, l'action politique n'est que la paradoxale irruption publique et concertée d'individus qui sont, en tant que tels, impuissants par définition. C'est pourquoi il est possible de dire que le moment où ces dix hommes ont commencé à se compter a été le premier signe de ruine de leur potentialité. 


\section{NOTES}

1. Condition de l'homme moderne (dorénavant CHM), Calmann-Lévy, 1983, Paris ; « Sur la violence », in Du mensonge à la violence. Essais de politique contemporaine, Calmann-Lévy, 1972, Paris (dorénavant $M V$ ).

2. Honig, Bonnie : "Arendt's Account of Action and Authority", in Political Theory and the Displacement of Politics, Cornell University Press, 1993, Ithaca and London.

3. $M V$, p. 144.

4. CHM, p. 259, c'est moi qui souligne.

5. CHM, p. 260.

6. CHM, p. 251.

7. MV, p. 151 ; Crises of the Republic, Harcourt Brace Jovanovich, San Diego - New York - London 1972, p. 149.

8. Voir par exemple $M V$, p. 141, aussi p. 151.

9. $M V$, p. 157.

10. Hilb, Claudia, «Violencia y política en la obra de Hannah Arendt», in Postdata, $n^{\circ} 6$, juillet 2000, Buenos Aires, p. 81.

11. Voir « Sur la violence », in MV, op. cit., p. 142, 153.

12. CHM, p. 260, c'est moi qui souligne.

13. Rancière, Jacques : La Mésentente. Politique et philosophie, Galilée, 1995, Paris.

14. Tassin, Étienne (1994) : «Pouvoir, autorité et violence. La critique arendtienne de la domination ", in J.-H. Go dard et B. Manille (sir.) : Le Pouvoir, Brin - Intégrale, 1994, Paris, pp. 285-286, c'est moi qui souligne.

15. La force, c'est l'«énergie qui se libère au cours de mouvements physiques ou sociaux», de sorte qu'on parle de la force des choses, les «forces de la nature» ou celle des «circonstances», MVp. 145.

16. $M V$, p. 142 , c'est moi qui souligne

17. $M V$, p. 149, c'est moi qui souligne (traduction modifiée).

18. $M V$, pp. 148-149, c'est moi qui souligne.

19. $M V$, pp. 150-151, j'ai modifié la traduction. Cela ne veut évidemment pas dire que la police secrète soit une source de pouvoir, mais que le chef totalitaire n'est pas seul, qu'il y a un certain nombre de gens qui l'accompagnent en portant les armes, en surveillant le peuple, etc.

20. Tel qu'Arendt l'indique, d'une part, avec l'exemple de David et Goliath, « dans un combat singulier [contest] ce n'est pas la puissance, c'est la force qui décide » et, d'autre part, avec l'exemple de résistance passive à la Gandhi : «puisque l'on ne peut s'y opposer par une lutte [ fighting] entraînant défaite ou victoire ». Tandis que dans un conflit singulier de force on dit que « $\mathrm{A}$ » confronte « $\mathrm{B}$ » et que dans un conflit singulier violent on dit que « $\mathrm{A}$ » et « $\mathrm{B}$ » confrontent à l'aide de l'instrument « $C$ », pour ce qui concerne les conflits de pouvoir, dont on pourrait dire qu'ils sont pluriels ou tout simplement "politiques", il n'existe pas une telle chose comme pouvoir-A versus pouvoir-B, il reste toujours un espace « entre " qui n'est pas définissable en termes de A, B ou C.

21. Nul doute, dans un hypothétique affrontement du pouvoir et de la pure violence, la victoire de celle-ci est inévitable. C'est pourquoi Arendt croit que si, au lieu de l'Angleterre, la résistance non violente mais puissante de Gandhi s'était opposée à la Russie de Staline ou à l'Allemagne de Hitler (des régimes totalitaires où le pouvoir est à peu près détruit), « elle ne se serait pas 
terminée par la décolonisation, mais bien par les massacres et la soumission.». Cf. "Sur la violence ", op. tic. p. 154, aussi pp. 148-149.

22. H. Arendt, « Compréhension et politique », in La Philosophie de l'existence et autres essais, Payot, 2000, Paris, pp. 210-212.

23. CHM, pp. 250-251.

24. S'ouvre ici la question du rapport entre politique et histoire, sujet important chez Arendt et qui mérite une approche particulière qui ne peut être abordée dans les limites de cet article.

25. C'est pourquoi les justifications impliquent une « limitation politique » à la violence. Voir $O R$, p. 19. Voir aussi MV., p. 155.

26. Voir Hilb, op. cit., pp. 81-84 et 87 ; aussi MV, par exemple pp. 180-181 et 183, où Arendt soutient que «la violence est payante » mais qu'elle paye indifféremment [indiscriminately], sans critère (voir p. 180, la traduction française sur ce point est malheureusement inexacte), puisqu'on est loin de la maîtrise propre à l'homo faber qui domine et transforme les matériaux par la violence.

27. «Le pouvoir corrompt sans doute lorsque les faibles se liguent pour ruiner les forts, mais pas avant. » (CHM, p. 264, j'ai modifié la traduction qui omettait l'expression " pour ruiner » [in order to ruin]).

28. Arendt a nommé «effet de boomerang " ce type d'expérience où un pouvoir violent envers l'extérieur se détruit lui-même à l'intérieur et dont l'exemple classique est l'impérialisme. "On peut obtenir la victoire en se servant de la violence comme d'un substitut du pouvoir, mais le prix qu'il faut payer est très élevé ; car il n'est pas payé seulement par le vaincu, mais également par le vainqueur, qui voit s'affaiblir son propre pouvoir. »Cet effet fut constaté dans le mode de répression utilisé par le gouvernement des États-Unis contre les étudiants pendant les événements de 1968 et au milieu de la guerre du Vietnam. L'Angleterre, en revanche, sut éviter de payer ce prix en liberté intérieure lorsqu'elle fut confrontée à la résistance de Gandhi, MV pp. 154-155. Cette question est aussi bien développée dans le deuxième volume de Les Origines du totalitarisme, consacré au sujet de l'impérialisme.

29. OR, p. 151.

30. Ce problème est présent chez Arendt par exemple dans CHM : "Dès que nous voulons dire qui est quelqu'un notre vocabulaire même nous entraîne à dire ce qu'il est » (p. 238).

\section{AUTEUR}

\section{LUCAS G. MARTÍN}

Doctorant à l'Université de Paris 7 - Denis Diderot. Programme Saint-Exupéry MECT - Ambassade de France (Argentine) 